\title{
DEMICONTINUITY, HEMICONTINUITY AND MONOTONICITY. II
}

\author{
BY TOSIO KATO ${ }^{1}$
}

Communicated by F. Browder, June 28, 1967

In the previous paper [6] with the same title, the writer proved that a (nonlinear) monotonic operator $G$ from a Banach space $X$ to the adjoint space $X^{*}$ is demicontinuous if and only if it is hemicontinuous and locally bounded, under a certain mild assumption on $D(G)$. (For similar results see also Browder [3].) In the present note we shall show that if $D(G)$ is an open set, the assumption of local boundedness is redundant so that hemicontinuity and demicontinuity are equivalent. Furthermore, we shall show that a similar result holds for a more general class of operators, including accretive operators in $X$ where $X^{*}$ is uniformly convex.

In what follows we consider (real or complex) Banach spaces $X, Y$ and (nonlinear) operators $F, G$ such that ( $D$ and $R$ denoting the domain and range, respectively) $D(F)=X, R(F) \subset Y, D(G) \subset X$, $R(G) \subset Y^{*}$.

Definition 1. $G$ is said to be $F$-monotonic if

$$
\operatorname{Re}(F(x-y), G x-G y) \geqq 0, \quad x, y \in D(G),
$$

where $($,$) denotes the pairing between Y$ and $Y^{*}$.

Definition 2. Let $u \in D(G)$. $G$ is said to be

(a) demicontinuous at $u$ if $u_{n} \in D(G), n=1,2, \cdots$, and $u_{n} \rightarrow u$ as $n \rightarrow \infty$ imply $G u_{n} \rightarrow G u$ (here and in what follows $\rightarrow$ and $\rightarrow$ denote strong and weak* convergence, respectively);

(b) locally bounded at $u$ if the conditions in (a) imply that $\left\|G u_{n}\right\|$ is bounded as $n \rightarrow \infty$;

(c) hemicontinuous at $u$ if $v \in X, t_{n}>0, n=1,2, \cdots, t_{n} \rightarrow 0$ and $u+t_{n} v \in D(G)$ imply $G\left(u+t_{n} v\right) \rightarrow G u$;

(d) locally hemibounded at $u$ if the conditions in (c) imply that $\left\|G\left(u+t_{n} v\right)\right\|$ is bounded as $n \rightarrow \infty$.

Obviously we have the implications

$$
\text { (a) } \stackrel{\text { (b) }}{\gtrless} \text { (c) } \gg \text { (d). }
$$

1 This work was partly supported by Air Force OSR Grant 553-64. 
EXAMPLE 1. Let $Y=X$ and $F=1$ (the identity operator in $X$ ). Then the $F$-monotonicity of $G$ means that $G$ is monotonic in the usual sense of Minty [8] and Browder [2].

Example 2. Assume that $X$ is reflexive and set $Y=X^{*}$. Further assume that $X^{*}$ is strictly convex. Let $F: X \rightarrow X^{*}$ be the duality map, which is well defined if we assume that $\|F x\|=\|x\|$ (see Beurling and Livingston [1], Browder [4]). In this case the $F$-monotonicity of $G$ (which has range space $Y^{*}=X$ ) means that $G$ is accretive in the sense of Browder [5]. We note that here $F$ is itself a monotonic operator in the sense of Example 1. Since $F$ is also known to be demicontinuous and coercive (see Browder [5]), it follows from a theorem of [8] and [2] that $F$ is onto $X^{*}$. We note also that $F$ is uniformly continuous (in the strong topologies) in any bounded subset of $X$ if $X^{*}$ is uniformly convex (see e.g. Kato [7]).

Theorem. Assume that

$\left(1^{\circ}\right) F$ is positive-homogeneous: $F(t x)=t F(x)$ for $t>0$;

$\left(2^{\circ}\right) \quad F$ is onto $Y: R(F)=Y$;

$\left(3^{\circ}\right) F$ is uniformly continuous in the closed unit ball of $X$ (in the strong topologies);

$\left(4^{\circ}\right) D(G)$ is open in $X$;

$\left(5^{\circ}\right) G$ is $F$-monotonic.

Then

(i) $G$ is locally bounded at $u \in D(G)$ if and only if it is locally hemibounded at $u$;

(ii) $G$ is demicontinuous at $u \in D(G)$ if and only if it is hemicontinuous at $u$.

In view of Examples 1, 2 given above, the following corollary is a direct consequence of the theorem.

Corollary. Demicontinuity and hemicontinuity are equivalent for $G$ in each of the following cases.

(a) $G$ is a monotonic operator with $D(G)$ open in $X$ and $R(G) \subset X^{*}$, $X$ being an arbitrary Banach space.

(b) $G$ is an accretive operator in $X$ with $D(G)$ open, $X^{*}$ being assumed to be uniformly convex.

Proof of the Theorem. (i) Since local boundedness at $u$ implies local hemiboundedness at $u$, it suffices to prove the converse implication. To this end, it suffices to show that $u_{n} \rightarrow u$ and $\left\|G u_{n}\right\|=r_{n} \rightarrow \infty$ lead to a contradiction if $G$ is locally hemibounded at $u \in D(G)$.

For each $s>0$ let $\phi(s)$ be the supremum of $\|F x-F y\|$ for $\|x\| \leqq 1$, $\|y\| \leqq 1$, and $\|x-y\| \leqq s$. Since by $\left(3^{\circ}\right) F$ is uniformly continuous in 
the unit ball of $X, \phi(s)$ is nondecreasing in $s$ and $\phi(s) \rightarrow 0$ as $s \rightarrow 0$. Furthermore, $\phi(s)<\infty$ for all $s>0$ in virtue of positive-homogeneity $\left(1^{\circ}\right)$. Thus

$$
\|F x-F y\| \leqq \phi(\|x-y\|) \quad \text { if } \quad\|x\| \leqq 1,\|y\| \leqq 1 .
$$

Set

$$
t_{n}=\operatorname{Max}\left[\left(1 / r_{n}\right),\left\|u_{n}-u\right\|{ }^{1 / 2}, \phi\left(\left\|u_{n}-u\right\|\right)^{1 / 2}\right]
$$

so that

$$
t_{n}>0, \quad t_{n} r_{n} \geqq 1, \quad\left\|u_{n}-u\right\| \leqq t_{n}^{2}, \quad \phi\left(\left\|u_{n}-u\right\|\right) \leqq t_{n}^{2} .
$$

Since $r_{n} \rightarrow \infty$ and $\left\|u_{n}-u\right\| \rightarrow 0$ by hypothesis, $\phi\left(\left\|u_{n}-u\right\|\right) \rightarrow 0$ too and

$$
t_{n} \rightarrow 0 \text { as } n \rightarrow \infty \text {. }
$$

Let $v \in X$ and $w_{n}=u+t_{n} v$. Since $u \in D(G)$ and $D(G)$ is open, $w_{n} \in D(G)$ for sufficiently large $n$. Thus we have by $\left(1^{\circ}\right)$ and $\left(5^{\circ}\right)$

$$
\begin{aligned}
\operatorname{Re}\left(F v, G u_{n}\right) & \leqq t_{n}^{-1} \operatorname{Re}\left(F\left(w_{n}-u_{n}\right), G w_{n}\right) \\
& +t_{n}^{-1} \operatorname{Re}\left(F\left(t_{n} v\right)-F\left(w_{n}-u_{n}\right), G u_{n}\right) .
\end{aligned}
$$

Let us estimate the right member of (5). First we note that $t_{n}^{-1}\left(w_{n}-u_{n}\right)=v-t_{n}^{-1}\left(u_{n}-u\right) \rightarrow v$ as $n \rightarrow \infty$ for $t_{n}^{-1}\left\|u_{n}-u\right\| \leqq t_{n}^{-1} \rightarrow 0$ by (3) and (4). Since $F$ is continuous by $\left(1^{\circ}\right)$ and $\left(3^{\circ}\right)$, we have $t_{n}^{-1} F\left(w_{n}-u_{n}\right)$ $=F\left(t_{n}^{-1}\left(w_{n}-u_{n}\right)\right) \rightarrow F v$. Since $\left\|G w_{n}\right\|=\left\|G\left(u+t_{n} v\right)\right\|$ is bounded as $n \rightarrow \infty$ by the assumed local hemiboundedness at $u$ of $G$, the first term on the right of (5) is thus bounded as $n \rightarrow \infty$.

To estimate the second term, we note that both $t_{n} v$ and $w_{n}-u_{n}$ tend to zero and hence belong to the unit ball of $X$ for sufficiently large $n$. Hence $\left\|F\left(t_{n} v\right)-F\left(w_{n}-u_{n}\right)\right\| \leqq \phi\left(\left\|t_{n} v-w_{n}+u_{n}\right\|\right)=\phi\left(\left\|u_{n}-u\right\|\right)$ $\leqq t_{n}^{2}$ by (1) and (3), so that the second term on the right of (5) is majorized by $t_{n}\left\|G u_{n}\right\|=t_{n} r_{n}$.

Thus we have the estimate

$$
\operatorname{Re}\left(F v, G u_{n}\right) \leqq C+t_{n} r_{n},
$$

where $C$ may depend on $v$ but not on $n$. Dividing (6) by $t_{n} r_{n}$ and noting that $t_{n} r_{n} \geqq 1$ by (3), we obtain

$$
\limsup _{n \rightarrow \infty} \operatorname{Re}\left(F v,\left(t_{n} r_{n}\right)^{-1} G u_{n}\right)<\infty .
$$

Since $F$ is onto $Y$ by $\left(2^{\circ}\right), y=F v$ in (7) can be any element of $Y$. Replacing $y$ by $-y$ (and by $\pm i y$ if $Y$ is complex), we see that $\left(y,\left(t_{n} r_{n}\right)^{-1} G u_{n}\right)$ is bounded as $n \rightarrow \infty$ for every $y \in Y$. But this is a 
contradiction to the principle of uniform boundedness, for $\left\|\left(t_{n} r_{n}\right)^{-1} G u_{n}\right\|=t_{n}^{-1} \rightarrow \infty$.

(ii) Again it suffices to show that $G$ is demicontinuous at $u \in D(G)$ if it is hemicontinuous at $u$. Let $u_{n} \rightarrow u$; we have to show that $G u_{n} \rightarrow G u$. Since hemicontinuity at $u$ implies local hemiboundedness at $u, G$ is locally bounded at $u$ by (i) just proved. Thus $r_{n}=\left\|G u_{n}\right\|$ is bounded as $n \rightarrow \infty$.

Now we set

$$
t_{n}=\operatorname{Max}\left[\left\|u_{n}-u\right\| 1 / 2, \phi\left(\left\|u_{n}-u\right\|\right)^{1 / 2}\right],
$$

so that (3) and (4) are again true except $t_{n} r_{n} \geqq 1$. With $w_{n}=u+t_{n} v$, we have again the inequality (5). Since $t_{n}^{-1} F\left(w_{n}-u_{n}\right) \rightarrow F v$ as before and since $G w_{n}=G\left(u+t_{n} v\right) \rightarrow G u$ by the hemicontinuity of $G$ at $u$, the first term on the right of (5) tends to $\operatorname{Re}(F v, G u)$. The second term is majorized by $t_{n} r_{n}$ as before. Since $r_{n}$ is now bounded, this term tends to zero. Thus we have

$$
\limsup _{n \rightarrow \infty} \operatorname{Re}\left(F v, G u_{n}-G u\right) \leqq 0 .
$$

Since $F v=y$ may be an arbitrary element of $Y$, it follows by an argument similar to the one used in (i) that $\lim \sup \left|\left(y, G u_{n}-G u\right)\right|=0$ for every $y \in Y$. Thus $G u_{n} \rightarrow G u$.

\section{BIBLIOGRAPHY}

1. A. Beurling and A. E. Livingston, $A$ theorem on duality mappings in Banach spaces, Ark. Mat. 4 (1962), 405-411.

2. F. E. Browder, Nonlinear elliptic boundary value problems, Bull. Amer. Math. Soc. 69 (1963), 862-874.

3. - Continuity properties of monotone nonlinear operators in Banach spaces, Bull. Amer. Math. Soc. 70 (1964), 551-553.

4. - On a theorem of Beurling and Livingston, Canad. J. Math. 17 (1965), $367-372$.

5. - Nonlinear accretive operators in Banach spaces, Bull. Amer. Math. Soc. 73 (1967), 470-476.

6. T. Kato, Demicontinuity, hemicontinuity and monotonicity, Bull. Amer. Math. Soc. 70 (1964), 548-550.

7. ——, Nonlinear semigroups and evolution equations, J. Math. Soc. Japan. (to appear).

8. G. J. Minty, On a "monotonicity" method for the solution of nonlinear equations in Banach spaces, Proc. Nat. Acad. Sci. 50 (1963), 1038-1041.

University of California, Berkeley 